\title{
Anomalous Blueshift in Vertically Coupled InAs/GaAs Quantum Dots Using InGaAs Strain-Reducing Layers
}

\author{
M. J. da Silva and A. A. Quivy \\ Instituto de Física da Universidade de São Paulo, \\ Laboratório de Novos Materiais Semicondutores \\ CP 66318, 05315-970 São Paulo, SP, Brazil
}

Received on 23 April, 2001

\begin{abstract}
The vertical coupling of InAs quantum dots and their capping with $\operatorname{In}_{0.1} \mathrm{Ga}_{0.9}$ As layers were investigated in order to shift the optical emission of the structures toward longer wavelengths. We observed that both ways can not be used simultaneously by just replacing part of the usual GaAs spacer layer by $\mathrm{In}_{0.1} \mathrm{Ga}_{0.9}$ As because the intermixing of $\mathrm{Ga}$ and In atoms is enhanced in stacked layers and causes a blueshift of the emission instead of the expected redshift.
\end{abstract}

\section{Introduction}

The study of low-dimensional semiconductor heterostructures is one of the most important topics in Solid-State Physics as a consequence of their application to optoelectronic devices like light-emitting diodes and lasers. A problem of current interest in this field is the possibility of extending the optical-emission range of GaAs-based devices up to $1.3 \mu \mathrm{m}$, which is a point of minimal attenuation of the optical fibers. InGaAs/GaAs quantum wells (QWs) cannot be used to reach the required range of wavelengths because of the limitations imposed by the two-dimensional (2D) strained growth. Recently, however, InAs/GaAs quantum dots (QDs) grown by molecular beam epitaxy (MBE) were pointed out as a reliable manner to reach this goal. When a thin InAs layer is deposited on a GaAs(001) substrate, the initial growth proceeds under 2D strained conditions and the elastic energy of the system can be relaxed by forming small coherent InAs islands at the surface when the thickness of the epitaxial layer slightly exceeds a critical value (1.6 MLs). When these islands are covered by GaAs, they act as quantum dots, exhibiting a three-dimensional (3D) confining potential, and emit within the 1.0-1.2 $\mu \mathrm{m}$ range at room-temperature. Two approaches have been suggested to extend their emission up to $1.3 \mu \mathrm{m}$. The first one is the vertical coupling of closely-stacked layers of QDs (separated by GaAs layers) that takes advantage of the natural tendency of the islands to align vertically [1] in order to produce an effective potential where the electronic levels are redshifted with respect to the ones of a single layer of QDs. This vertical ordering has the additional benefit of increasing the size uniformity of the islands. The second method uses a single layer of InAs/GaAs QDs capped by a InGaAs film that acts as a strain-reducing layer [2] and promotes a redshift of the electronic levels. Both methods have a major drawback. In order to reach the $1.3 \mu \mathrm{m}$ region, the vertical coupling requires a large number of QD layers that considerably increases the concentration of non-radiative centers. The capping of a single QD layer with InGaAs is efficient to tune the emission toward longer wavelengths, but the wide size distribution of the QDs in a single layer broadens the luminescence spectra (up to $90 \mathrm{meV}$ ) and is disadvantageous to most of the highperformance optical devices.

In this work, we investigated the possibility of combining both approaches to produce vertically coupled InAs QDs separated by thin spacer layers where part of the GaAs material was substituted by InGaAs. In this way, we expected to take advantage of the better homogenization of the structures provided by the stacking procedure and of the faster tuning of the emission by the InGaAs strain-reducing layers in order to obtain samples emitting around $1.3 \mu \mathrm{m}$ with a reduced number of interfaces and a narrower line width.

\section{Samples and experimental setup}

All the samples discussed here were grown in a Mod. Gen II MBE system on GaAs(001) epi-ready substrates. A $0.2 \mu \mathrm{m}$-thick GaAs buffer layer was deposited at $580^{\circ} \mathrm{C}$ before the growth of any of the structures that will be discussed in their respective section. The InAs and InGaAs layers, as well as the GaAs spacer and cap 
layers were deposited at $500^{\circ} \mathrm{C}$ to avoid In revaporation. The Ga, In and As fluxes were supplied at a rate of 1.0, 0.05 and 2.5MLs/s, respectively, except when specified differently. The samples were optically characterized by photoluminescence (PL) at $2 \mathrm{~K}$ and at room temperature, and structural measurements were done by

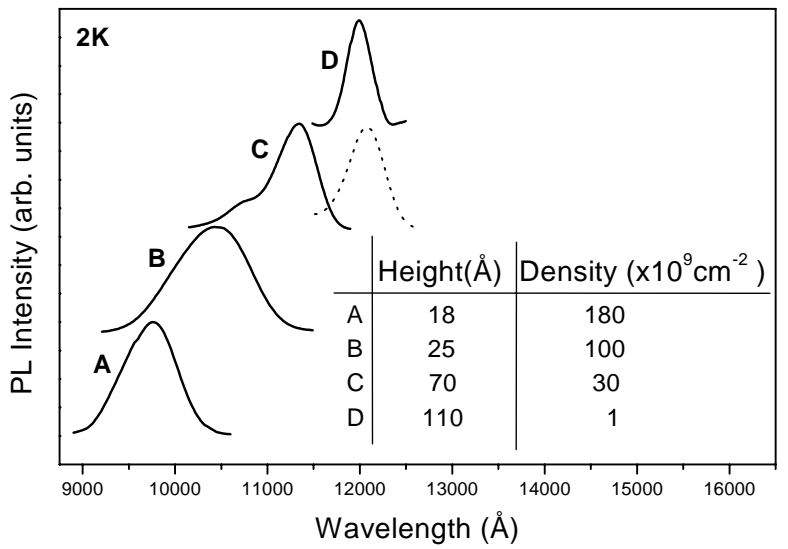

Figure 1. 2K PL spectra of a single 2.4ML-thick InAs QD layer grown with a In delivery rate of A) $0.1 \mathrm{ML} / \mathrm{s}$, B) $0.08 \mathrm{ML} / \mathrm{s}$, C) $0.05 \mathrm{ML} / \mathrm{s}$ and D) $0.005 \mathrm{ML} / \mathrm{s}$. The inset shows the height and density of the QDs as measured by atomic force microscopy on similar samples without the $300 \AA$-thick GaAs cap layer.

cross-section transmission electron microscopy (XTEM).

\section{Results and discussion}

The simplest way to redshift the optical emission of the QDs is growing structures with a larger size. That can be achieved, within some limits, by modifying the diffusion length of the In adatoms that mainly depends on the In delivery rate, the $\mathrm{V} / \mathrm{III}$ flux ratio and the substrate temperature used to deposit the InAs film. Figure 1 shows the redshift obtained in the first set of samples where the InAs growth rate was varied. The main drawback of this approach is that, when the size of the structures becomes larger, their density is reduced and is no longer adequate for optoelectronic applications.

A second way to redshift the optical emission of such structures is to reduce the strain of the system resulting from the lattice mismatch between InAs and GaAs. Figure 2 shows the PL spectra of the second set of samples where a 2.4ML-thick InAs QD layer was capped by $\mathrm{In}_{0.1} \mathrm{Ga}_{0.9} \mathrm{As}$ in order to reduce the strain of the islands. Each sample had a InGaAs cap of different thickness and received an extra layer of GaAs to complete a total cap-layer thickness of $300 \AA$. It is clear from figure 2 that the strain reduction achieved by the $\mathrm{In}_{0.1} \mathrm{Ga}_{0.9}$ As layer is very effective in redshifting the optical emission and most of the effect is obtained for a $\mathrm{In}_{0.1} \mathrm{Ga}_{0.9}$ As thickness around $25 \AA$ that corresponds to slightly more than half of the height of these buried QDs [3].

Based on the experimental results of both previous sets of samples, a third set was grown where several QD layers were stacked and capped according to sample $\mathrm{G}$. A total spacer layer of $50 \AA$ was used between two

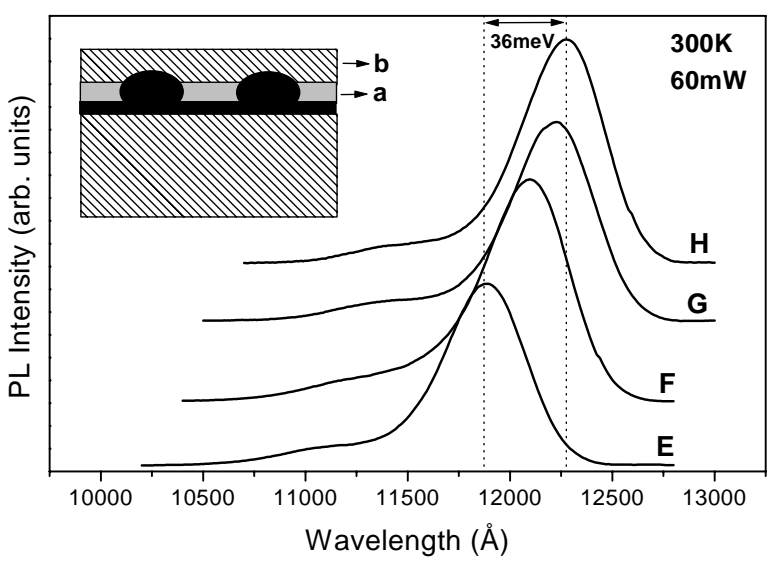

Figure 2. 300K PL spectra of a single 2.4ML-thick InAs QD layer capped by a $\mathrm{In}_{0.1} \mathrm{Ga}_{0.9}$ As layer with a thickness a equal to E) $0 \AA \mathrm{F}) 10 \AA \mathrm{G}) 25 \AA$ and $\mathrm{H}) 50 \AA$. Finally, a GaAs layer was deposited to complete the total cap-layer thickness of $300 \AA(=a+b)$.

consecutive QD layers since that specific separation previously provided a good electronic coupling for stacked QDs layers grown in the same conditions but separated by a GaAs layer [3]. It can be seen in figure 3 that the three samples containing stacked layers (J-L) are blueshifted with respect to the reference sample (I), unlike expected, but there exists a systematic redshift of the stacked samples with respect to sample $\mathbf{J}$ when the number of stacked layers increases.

The behavior observed in figure 3 can be qualitatively understood considering the following model. An uncapped InAs QD has an equilibrium size and composition that minimize the surface free energy of the system for specific strain and growth conditions. When the island is capped, an additional stress is imposed which modifies the equilibrium condition of the new system. To recover the minimal-energy condition, an intermixing effect takes place during the growth of the cap layer and In atoms of the island are substituted by Ga atoms of the growing cap layer. This phenomenon leads to a smaller and Ga-rich island that yields a blueshift of the optical emission with respect to an uncapped QD [4]. The shrinking of capped InAs QDs can be clearly observed in cross-section TEM images that show islands with about half of the vertical size of free-standing islands grown in the same conditions (figure 4 ). When several QD layers are stacked, the equilibrium condition of the buried island is further modified as a consequence of the penetrating strain field coming from the 
upper QD layer, as confirmed in figure $4 \mathrm{~b}$. When the InGaAs and GaAs cap layers of the reference sample are grown, kinetic and thermodynamic considerations during deposition dictate the strength and extent of the intermixing in order to minimize the free energy of the system, leading to spectrum I of figure 3. However, when a second QD layer is grown atop $(\mathbf{J})$, the strain

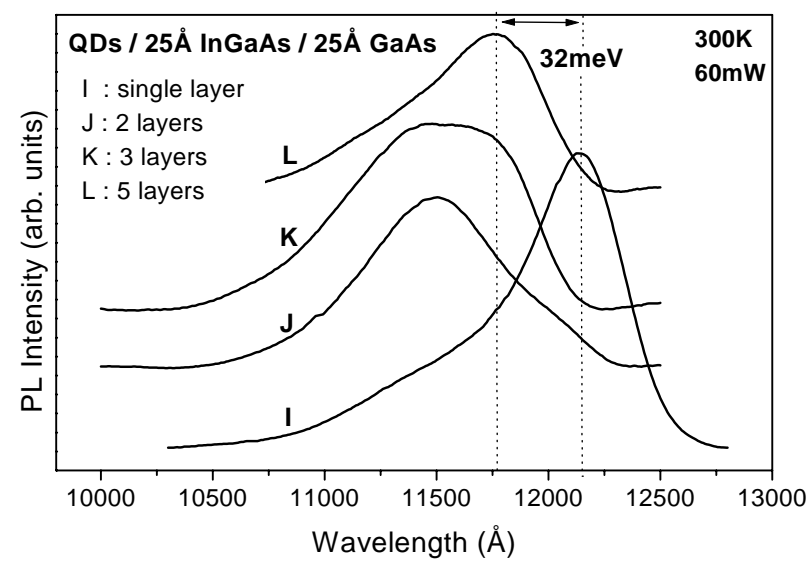

Figure 3. 300K PL spectra of stacked QD layers grown according to sample $\mathrm{G}$. The number of stacked QD layers in each sample is I) 1, J) $2, \mathrm{~K}) 3$ and L) 5 .
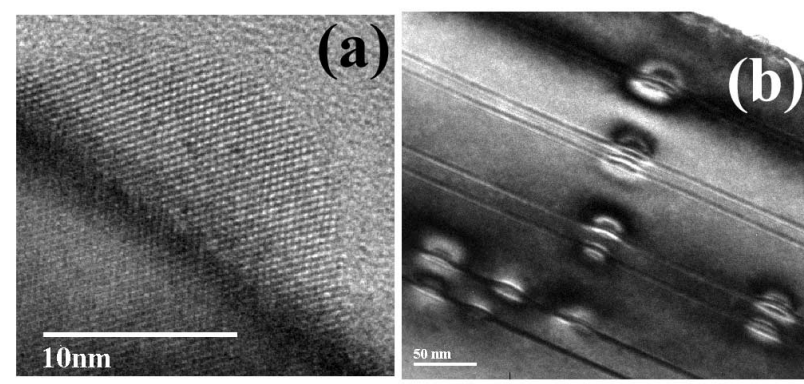

Figure 4. (a) High-resolution TEM image of a free-standing InAs QD grown in the same conditions as sample C (b) Dark field (400) TEM image of four pairs of QD layers separated by $500 \AA$ of GaAs. The GaAs spacer between each QD layer of a pair is 50,100,150 and $200 \AA$ (from top to bottom). The overlap of the strain field from each QD layer of a pair is visible.

configuration of the system is modified and turns to be the driving force to induce further intermixing in the underneath QD layer, yielding even smaller and more Ga-rich islands. Since the top QD layer is also affected by the strain field of the lower layer (the QDs are slightly larger) and the intermixing is stronger for larger QDs, the whole system will have its emission blueshifted with respect to the reference sample. However, when the number of stacked layers increases, the vertical electronic coupling becomes more effective and a redshift of the emission is observed $(\mathrm{K}-\mathrm{L})$.

\section{Conclusion}

Using PL measurements, we observed that stacks of In As QD layers alternated with InGaAs strain-reducing spacers are not able to shift the usual emission of the structures toward $1.3 \mu \mathrm{m}$ as would be expected. The blueshift detected in the PL spectra indicates a relevant intermixing during the capping and stacking processes. The driving force for the exchange of In atoms of the buried QDs by Ga atoms of the cap layer seems to be related to the penetration of the strain field coming from the upper QD layers of the stack. If both methods have to be used together in order to shift the optical emission of the samples toward longer wavelengths, the intermixing has to be reduced. Since the phenomenon is thermally activated [5], one possible way to achieve that is by kinetically limiting the process using a lower substrate temperature.

\section{Acknowledgments}

We are grateful to FAPESP for financial support (grants 99/08979-7, 99/01225-7, 98/14489-0), to the "Laboratório de Filmes Finos do IFUSP" for the SPM facility (FAPESP proc. 95/05651-0) and to the "Laboratório de Microscopia Eletrônica (LME/LNLS)" for the TEM facility.

\section{References}

[1] G. S. Solomon, J. A. Trezza, A. F. Marshall, J. S. Harris Jr, Appl. Phys. Lett., 76, 952 (1996).

[2] Y. Nakata, K. Mukai, M. Sugawara, K. Ohtsubo, H. Ishikawa, N. Yokoyama, J. Crys. Growth, 208, 93 (2000).

[3] M. J. da Silva, A. A. Quivy, 10th Brazilian Workshop on Semiconductor Physics, April, 22-27 2001, Guarujá, SP, Brazil.

[4] O. G. Schmidt, K. Eberl, Phys. Rev. B, 61, 13721 (2000).

[5] M. Ustinov, A. Yu. Egorov, A. R. Kovsh, A. E. Zhukov, M. V. Maximov, A. F. Tsatsulnikov, N.Yu. Gordeev, S. V. Zaitsev, Yu. M. Shernyakov, N. A. Bert, P. S. Kopev, Zh. I. Alferov, N. N. Ledentsov, J. Bohrer, D. Bimberg, A.O. Kosogov, P. Werner, U. Gosele, J. Crys. Growth, 175, 689 (1997). 